\title{
EXPRESSION AND PURIFICATION CAPSID PROTEINS VP0, VP1 AND VP3 OF FOOT AND MOUTH DISEASE VIRUS TYPE O IN ESCHERICHIA COLI
}

\author{
Nguyen Hoang Duong ${ }^{1, *}$, Chi-Ning Chuang ${ }^{3}$, Nguyen Phuong Hoa ${ }^{1}$, \\ Tran Thi Kim Dzung ${ }^{1}$, Le Thi Hong Minh ${ }^{2}$, Nguyen Mai Anh', \\ Nguyen Thi Kim Cuc ${ }^{2}$, Le Thu Ha ${ }^{1}$, Pham Viet Cuong ${ }^{1,2}$, Ting-Wang Fang ${ }^{3}$ \\ ${ }^{1}$ Mientrung Institute for Scientific Research, Vietnam Academy of Science and Technology \\ ${ }^{2}$ Institute of Marine Biochemistry, Vietnam Academy of Science and Technology \\ ${ }^{3}$ Institute of Molecular Biology, Sinica Academia, Taiwan \\ *Email: nhduong.misr@gmail.com
}

Received: 8 December 2015; Accepted for publication: 2 June 2016

\begin{abstract}
Foot and mouth disease virus (FMDV) causing infectious disease affects broadly clovenhoofed animals. It has 7 different serotypes. However, type $\mathrm{O}$ is the most prevalent in 3 founded types $(\mathrm{O}, \mathrm{A}$, Asia1) recurrence in Vietnam fields. The current vaccines used for FMDV is inactivated or attenuated forms. FMDV Vaccines were able to raise strong immune responses in host organisms, but still caused the safety concerns. Virus-like particles could be a new vaccine generation that fulfills the present questions. On the one hand, it can tackle the safety issues, on the other hand, it can reserve FMDV intrinsic form which will provoke high immunogenicity. The important initial step to make virus-like particles is expression of the capsid proteins of FMDV in appropriate system. So in this study we describe how to design, express and purify of all capsid proteins: VP0, VP1 and VP3 of O type FMDV isolated in Vietnam field using SUMO fusion expression system.
\end{abstract}

Keywords: FMDV, SUMO, type O, vaccine, VLPs.

\section{INTRODUCTION}

Foot and mouth disease (FMD) is endemic in many developing countries which mainly affects pigs, sheep, cattle and cloven-hoofed animals. FMD outbreaks cause huge economic losses through direct or indirect effects on agriculture, meat supply and tourism. Some big outbreaks were recorded around the world such as in the UK (2001), Italia (1993) or Taiwan (1997) [1]. In Vietnam, the FMD circulates every year mainly with O, A and Asia 1 types [2, 3]. Causative agent of FMD is FMD virus (FMDV) which exists at 7 types (O, A, C, Asia 1, SAT1, SAT2, SAT3) and more than 60 serotypes. 
FMDV, like other Picornaviruses, is structured by a positive-sense, single RNA genome in length of 8,4 kb including 5' untranslated region (5'UTR), 3' untranslated region (3'UTR) and a long coding region encoded for a precursor polyprotein which is then processed by virus-code proteases to form structure and non-structure proteins necessary for virus propagation [4]. In stage of forming virion, RNA is surrounded by capsid proteins as a particle with the size in range of $25-30 \mathrm{~nm}$. The capsid particle is composed of 60 copies of the protomers. Each protomer contains four structural proteins of VP1, VP2, VP3 and VP4, among which VP2 and VP4 were self-cleaved by VP0. While VP1, VP2 and VP3 are exposed on surface of the virus, VP4 is located internally. During viral maturation, 5 protomers are assembled into a pentamer, then 12 pentamers associate to each other to form a virus particle $[5,6]$.

Current FMD vaccines are produced by infecting baby hamster kidney-21 cells with virulent FMDV type. In some endemic areas, the vaccine commonly contains several types of virus selected from infected regions. Although the conventional inactivated FMDV vaccine shown effectively protect vaccinated animals in many areas, there are still some limitations and concerns of using conventional vaccine in emergency control programs. The FMD free countries seem do not welcome the use of inactivated vaccine due to the possibility of spreading waked virulent strains caused by any insufficient inactivation during vaccine production and vaccination [7]. On the another hand, the incapability of inducing sterile immunity (100\% protection) may allow viral replication in the epithelial surface following live virus administration [8]. Besides, there are other important shortcomings of current inactivated vaccines, including short shelf life, the need for adequate cold chain of formulated vaccines, and difficulties of certain serotypes and subtypes to grow well in cell culture for vaccine production [9]. Many attempts are used as newly approaches to overcome the limitation of the conventional vaccines such as subunit or DNA vaccines which have been developed [1]. The most important FMDV antigen, VP1 containing neutralizing epitopes of the virus which was used for immunization $[7,10]$, and VP1 vaccine showed protection animal from viral challenge [11]. Furthermore, the IgG-based chimeric protein, FMDV immunodominant epitopes, FMDV proteins/peptides expressed in plants and T-cell epitopes also were used for immunization of different susceptible animals with limited success [1]. Other groups created virus-like particles (VLP) from either P1-2A-3C or VP0 and VP1-2A-VP3 combination, using baculovirus expression systems $[12,13]$. Both cassettes could automatically be cleaved in several steps to form VP0, VP1 and VP3 and then self-assembled into VLP forms. To simplify VLP making procedures, in this study we designed and expressed VP0, VP1 and VP3 in E. coli cells separately under the control of the same T7 promotor. Moreover, the recombinant proteins were fusioned with SUMO (small-ubiquitin-related modifier) to enhance water solubility and postprotein translation conformation [14]. Six (6) his-tag sequence was also added at the upstream of SUMO fusion protein. The purified intrinsic recombinant proteins (VP0, VP1 and VP3) could be harvested using homemade SUMO protease and Ni resin. Soluble purified VP0, VP1 and VP3 are 3.fundamental step to form VLP particle.

\section{MATERIALS AND METHODS}

\subsection{Materials}

FMDV cDNA extracted from swine sample were collected from the endemic FMD outbreak in 2013 in Hanoi, Vietnam. 


\subsection{Methods}

\subsubsection{Design expression vector for VPO, VP1 and VP3}

In our previous work [15], we described successful cloning of VP0, VP1 and VP3 from cDNA of Vietnam FMDV type $\mathrm{O}$ isolated in. Based on those sequences, 3 primer pairs for VP0, VP1 and VP3 with overhang restriction recognition sequences for $S f o \mathrm{I}$ and $\mathrm{XhoI}$ at both ends were designed. The primers parameters were calculated with support from IDT online software (USA). In addition, upstream primer was added GGC encoded for Glycine making recognition site for SUMO protease (Lys-Lys) at the end of the SUMO. Downstream primer was added stop codon TTA. Sticky-end PCR method was used to ligate PCR products into expression vectors (as demonstrated in Fig. 1) [16]. The desired sequences were amplified in two different reactions using two different primer pairs. The obtained PCR products were mixed, denatured at $95{ }^{\circ} \mathrm{C}$ for 5 minutes and annealing at $65{ }^{\circ} \mathrm{C}$ for 10 minutes. Approximately $25 \%$ of the final product carried desirable protruding ends and was ready for ligation. To increase ligation efficiency between PCR products and expression vectors, the kinase reaction was performed for phosphorylation of 5'-ends of the PCR products. The vectors pHD-Amp-RecA and pHD-KanRecA were derived from pET32-Xa/LIC (Novagen) after some modifications. The expression vectors contain ampicilin or kanamycin resistant gene and encode for 6-His-SUMO-RecA fusion protein. Restriction sites for $S f o \mathrm{I}$ and $X h o \mathrm{I}$ were designed at two ends of RecA. RecA was then substituted by either VP0, VP3 or VP1 forming pHD-Amp-VP0, pHD-Amp-VP3 or pHD-KanVP1, respectively.

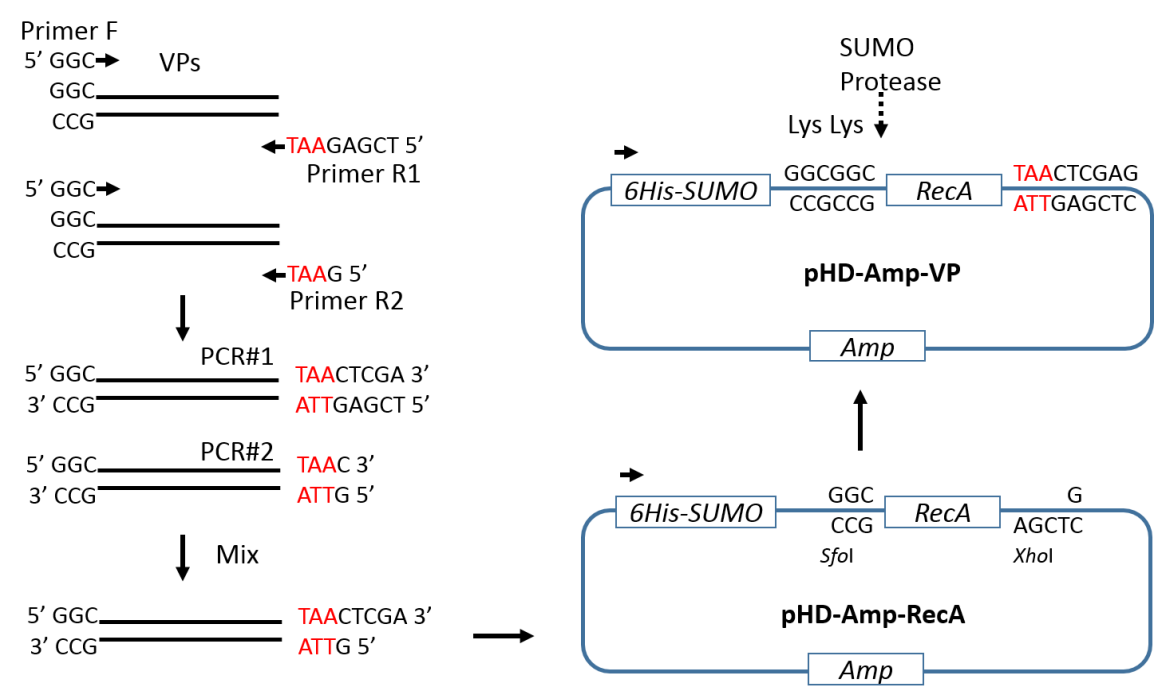

Figure 1. Vector construction for expression of FMDV capsid proteins: VP0, VP1 and VP3.

Our previous work showed that VPs recombinant proteins have aggregated together when they were separately expressed (data not shown). So in this study, 3 VPs genes were expressed in the same cells, VP0 expression cassette from pHD-Amp-VP0 was first genetically subcloned into pHD-Amp-VP3 vector, named pHD-Amp-VP3-VP0. For this purpose, VP0 expression cassette was amplified by using two primer pairs VP0-trans-F1/R2 and VP0-trans-F2/R1 overhanging ApaI and SphI (Tab. 1) using PCR sticky-end method. pHD-Kan-VP1 and pHDAmp-VP3-VP0 were simultaneously transformed into competent BL21 (DE3)-RIL E. coli cells 
(Stratagen, USA) under the selection of Kan, Amp and chloramphenicol at final concentration of 50,100 and $34 \mu \mathrm{g} / \mathrm{ml}$, respectively.

Table 1. Primer sequences used for amplification of VP0, VP1, VP3 and VP0 expression cassettes.

\begin{tabular}{|l|l|}
\hline \multicolumn{1}{|c|}{ Sequence } & \multicolumn{1}{c|}{ Name } \\
\hline 5' ggcACCACCTCCACAGGTGAG 3' & VP1-F \\
\hline 5' g ttaCAAAAGCTGTTTCACAGGTG 3' & VP1-R1 \\
\hline 5' tcgag ttaCAAAAGCTGTTTCACAGGTG 3' & VP1-R2 \\
\hline 5' ggcAACACTGGGAGCATCATTAAC 3' & VP0-F \\
\hline 5' g ttaTTCCTTGGAAGGGAACTC 3' & VP0-R1 \\
\hline 5' tcgag ttaTTCCTTGGAAGGGAACTC 3' & VP0-R2 \\
\hline 5' ggc GGGATCTTTCCCGTGGCGTG 3' & VP3-F \\
\hline 5' g ttaCTGCGTGCGAGCGTCAAc 3' & VP3-R1 \\
\hline 5' tcgag ttaCTGCGTGCGAGCGTCAAc 3' & VP3-R2 \\
\hline 5' ggcccGGAATGGTGCATGCAAGGAG 3' & VP0-Trans-R1 \\
\hline 5' cGGAATGGTGCATGCAAGGAG 3' & VP0-Trans-R2 \\
\hline 5' catgcGAACGTGGCGAGAAAGGAAG 3' & VP0-Trans-F1 \\
\hline 5' cGAACGTGGCGAGAAAGGAAG 3' & VP0-Trans-F2 \\
\hline
\end{tabular}

\subsubsection{Expression and solubilization of recombinant fusion proteins}

Successful transformants were cultured in $2 \mathrm{ml}$ LB broth containing Amp $(100 \mu \mathrm{g} / \mathrm{ml})$, Kan $(50 \mu \mathrm{g} / \mathrm{ml})$ and $1 \%$ glucose in $37^{\circ} \mathrm{C}$, incubator at $200 \mathrm{rpm} .10 \%$ overnight culture suspension was used to inoculate $50 \mathrm{ml}$ fresh LB medium supplemented with antibiotics and $1 \%$ glucose at $37{ }^{\circ} \mathrm{C}$. When $\mathrm{OD}_{600}$ reach value of 0.6 , IPTG (isopropyl-b-D-thiogalactopyranoside) was added at $1 \mathrm{mM}$ final concentration, whereas $1 \mathrm{ml}$ culture medium without IPTG was used as noninduced control. Cells were continuously grown at $20{ }^{\circ} \mathrm{C}$ for 20 hours. After the induction, cell lysates were harvested by using centrifugator at $5000 \mathrm{rpm}$ for 5 minutes at $4{ }^{\circ} \mathrm{C}$. Cell walls were disrupted in lysis buffer added $0.3 \mathrm{mg} / \mathrm{ml}$ lysozyme (Sigma) for 30 minutes at $4{ }^{\circ} \mathrm{C}$. Nuclei was continuously digested with benzonase (Invitrogen) for another 10 minutes and $\mathrm{NaCl}$ was adjusted to final concentration of $300 \mathrm{mM}$. The mixture was then sonicated 3 times, 10 seconds on/off and centrifuged at maximum speed at $4{ }^{\circ} \mathrm{C}$. Supernatant was collected and analyzed on $12 \%$ SDS-PAGE.

\subsubsection{Protein purification and SUMO cleaving}

The Ni-NTA Protein Purification System (Qiagen) was used to purify SUMO fusion histag proteins. Soluble mixture proteins were mixed with Ni-NTA resin with agitation at $4{ }^{\circ} \mathrm{C}$ for 1 hour. Mixture was went through equilibrated column. Flow-through solution was kept for affinity testing of the resin and recombinant proteins. Histag proteins binding to $\mathrm{Ni}$ resin were washed 3 times with NWB buffer $\mathrm{pH} 8(20 \mathrm{mM}$ Imidazole, $50 \mathrm{mM} \mathrm{NaH} 2 \mathrm{PO} 4.2 \mathrm{H} 2 \mathrm{O}, 500 \mathrm{mM}$ 
$\mathrm{NaCl})$ and eluted with NEB buffer (250 $\mathrm{mM}$ Imidazole, $50 \mathrm{mM} \mathrm{NaH} 2 \mathrm{PO} 4.2 \mathrm{H} 2 \mathrm{O}, 500 \mathrm{mM}$ $\mathrm{NaCl})$. The flow-through solution was tested on $12 \%$ SDS-PAGE.

Purified home-made SUMO protease [14] expressed by E. coli was used to cleave HisSUMO out of VP proteins (VP0, VP1 and VP3) at Lys-Lys site right before first amino acid codon of VP proteins resulting in authentic VP proteins. The reaction was carried on $4{ }^{\circ} \mathrm{C}$ for 4 hours. Digested proteins were concentrated using Vivaspin 20 centrifugal concentrator (GE Healthcare, USA) to remove unwanted high molecular weight proteins.

\subsubsection{Western blotting}

Western blotting (immunoblotting) is a commonly used method to detect specific antigens by its polyclonal or monoclonal antibodies. In this study, Western blotting was used to detect FMDV. Proteins was used for gel electrophoretic separation on $10 \%$ SDS-PAGE gels and transferred on to nitrocellulose or polyvinylidenedifluoride (PVDF) membrane by liquid blotting system (Invitrogen). Membrane was blocked with $5 \%$ milk in PBS-T and then was incubated with anti-FMDV primary antibodies at $4{ }^{\circ} \mathrm{C}$ for overnight (1:1000). The membrane was washed 4 times with PBS-T washing buffer. The membrane was next incubated with a secondary antibody conjugated with horseradish peroxidase (HRP) for 1 hour at RT (1:5000). After washing, luminescent substrate was added onto membrane to visualize the HRP activity. Signals released were detected on autoradiograph films.

\section{RESULTS AND DISCUSSION}

\subsection{Design expression vectors for VP0, VP1 and VP3}

Three pairs PCR products of VP0, VP1 and VP3 with the length of $\sim 861 \mathrm{bp}, \sim 639 \mathrm{bp}$ and $\sim 660 \mathrm{bp}$, respectively, were amplified using two separate PCR reactions with one forward primer and two reverse primers (Fig. 2, lane 1-6). In parallel, pHD-Amp-RecA and pHD-KanRecA were digested by $S f o \mathrm{I}$ and $\mathrm{XhoI}$ and purified by gel extraction (Invitrogen) (Fig. 2, lane 78). PCR products of each type were mixed and were 5'- end phosphorylated by T4 polynucleotide kinase to enhance ligation efficiency. While pHD-Amp was ligated with VP0 and VP3, pHD-Kan was ligated with VP1. The ligation mixtures were then transformed into JM109 competent cells. Colonies formed after overnight incubation were inoculated in LB medium containing Amp or Kan. Plasmid extraction and enzyme digestion were performed to confirm ligation process. Based on vector and gene sequences 2 restriction enzymes were picked which located on vector and the transgene. As shown in Fig. 2 (lane 9-26), all 6 randomly picked colonies brought suitable inserts of $V P O, V P 1$ or $V P 3$, with fragments with proper length after digestion with $E c o$ RI. This enzyme (EcoRI) has one cut on the inserts and one cut on the vector, so if cloning successful occurred, the digestion products would have 2 bands with the length of 630 and 5830 bp for pHD-Kan-VP0 (lane 9-14), 369 and 5869 bp for pHD-Kan-VP1 (lane 1520) and $535 \mathrm{bp}$ and $5875 \mathrm{bp}$ for pHD-Kan-VP3 (lane 21-26). Moreover, plasmids containing $V P O, V P 1$ and $V P 3$ were sequenced and results confirmed the successful cloning of $V P O, V P 1$ and $V P 3$ in expression vectors of pHD-Amp-VP0, pHD-Amp-VP3 and pHD-Kan-VP1 (data not shown). 


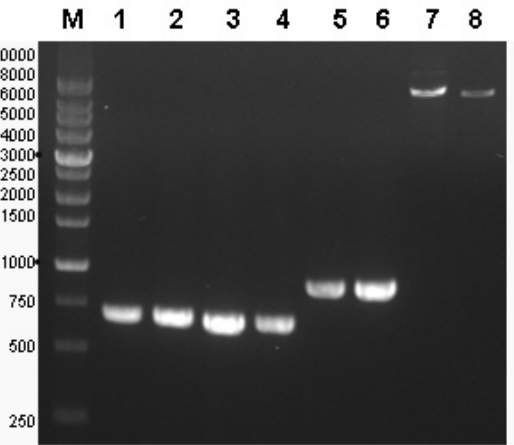

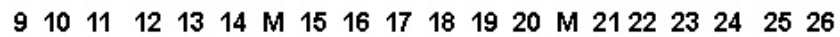

Figure 2. Cloning expression vectors for VP0, VP1 and VP3. Lane 1-2, 3-4, 5-6 are PCR products of VP3, VP1 and VP0, respectively, using 2 primer pairs (primer F/R1 and primer F/R2). VP0, VP1, VP3 were ligated into pHD-Amp (opened vector, lane 7) or pHD-Kan (opened vector, lane 8) then transformed

into JM109 cells. Six clones of each transformation were selected for plasmid extraction and EcoRI digestion. Lane 9-14, 15-20, 21-26 are plasmid digestions of pHD-Amp-VP0, pHD-Kan-VP1 and pHDAmp-VP3. M is $1 \mathrm{kbp}$ ladder marker (Invitrogen).

Next, VP0 and VP3 expression cassettes were linked together in one expression vector (pHD-Amp-VP3-VP0). This step helps reducing one antibiotic selection and backbone-gene burden for next expression step. For that purpose, sticky-end PCR method was used to prepare inserts. Two PCR reactions used with VP0-trans-F1/R2 primer pair and VP0-trans-F12/R1 primer pair to amplify VP0 expression cassette with the length of $\sim 1.8 \mathrm{kbp}$ (Fig. 3, lane 2-3). After 5'- end phosphorylation, denaturation and annealing, one fourth of the PCR mixture contained 2 suitable overhang of SphI and ApaI. Concurrently, pHD-Amp-VP3 was digested with SphI and ApaI and purified (Fig. 3, lane 1).

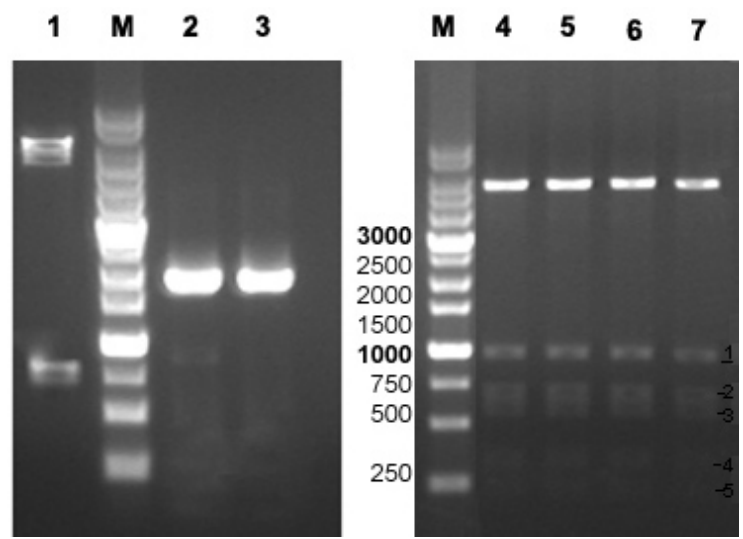

Figure 3. Construction of pHD-Amp-VP3-VP0. VP0 expression cassette was amplified using 2 primer pairs VP0-Trans-F1/R2 (lane 2) and VP0-Trans-F2/R1 (lane 3). pHD-Amp-VP3 was digested with SphI and ApaI (lane 1). Ligation product was transformed into JM109 competent cells. Four colonies were picked and cultured for plasmid extraction. Plasmids were cut by XhoI and EcoRI (lane 4-7). M is $1 \mathrm{~kb}$ ladder (Invitrogen).

Fragment about $6.5 \mathrm{kbp}$ was extracted from agarose gel. The ligation of VP0 expression cassette and the vector fragment of pHD-Amp-VP3 was performed as described in method with the same procedure mentioned above. Ligation was transformed into JM109 competent cells. 
Plasmid extraction and digestion with XhoI and EcoRI were analyzed on $1 \%$ agarose gel. As showed in Fig. 3 (lane 4-7), four randomly picked plasmids indicated as positive clones which were digested into 6 bands; 1 plasmid backbone ( $4924 \mathrm{bp}$ ) and 5 smaller fragments ( $879 \mathrm{bp}, 630$ bp, $535 \mathrm{bp}, 317 \mathrm{bp}, 211 \mathrm{bp}$ ) as the same as the hypothetic analysis using Vector_NTI software (the software analyze hypothetic digestion with indicated enzymes when was produced vector or gene sequences).

\subsection{Recombinant protein production}

Recombinant plasmids pHD-Amp-VP3-VP0 and pHD-Kan-VP1 were transformed into competent BL21 (DE3)-RIL E. coli cells. To express recombinant proteins, pHD-Amp-VP3VP0 and pHD-Kan-VP1 were transformed into competent BL21 (DE3)-RIL E. coli cells. Behind containing $\mathrm{T} 7$ polymerase, this type of cell also bring extra copies of the argU, ileY, and leuW tRNA genes. These genes encoded tRNAs that recognized the arginine codons AGA and AGG, the isoleucine codon AUA, and the leucine codon CUA, respectively. The CodonPlus-RIL strains encoded the tRNAs that most frequently restrict translation of heterologous proteins from organisms that have AT-rich genomes. For using this E. coli cell type, it will increase the expressing possibility of 3 transgenes: VP0, VP1 and VP3 which were derived from FMDV virus.

As shown in Fig. 4, expressed VP0, VP1 and VP3 fusions were water soluble proteins. The obvious difference between induction and non-induction lanes was shown (lane I and lane $\mathrm{S}$ ). The expression of VPs fusion proteins were demonstrated clearly after protein purification with the molecular weight of purified proteins were in the range from 45 to $52 \mathrm{kDa}$, conformity with predicted weights of $6 \mathrm{His}+\mathrm{SUMO}+\mathrm{VP} 1,6 \mathrm{His}+\mathrm{SUMO}+\mathrm{VP} 3$ and 6His+SUMO+VP0. Digestion of VPs fusion proteins with His-SUMO protease resulted in 3 lower bands with molecular weight of about $25 \mathrm{kDa}, 28 \mathrm{kDa}$ and $32 \mathrm{kDa}$ in according with VP1, VP3 and VP0, respectively. Furthermore, two other digestive by-products were 6His-SUMO with molecular weight of 18 $\mathrm{kDa}$ and His-SUMO protease with MW of $35 \mathrm{kDa}$ seen on SDS-PAGE after digestion. Concurrently, the same protein amount with SDS-PAGE was set up for western blotting with FMDV polyclonal antibody. The results clearly indicated the shift of VPs-fusion to VPs after His-SUMO protease digestion (Fig. 4). VP0, VP1 and VP3 from SDS-PAGE were directly cut off for amino acid sequencing analysis using mass spectrometry (MS). The results which were compared to online database have confirmed the correct expression of recombinant proteins of FMDV type $O$ (data not shown). This indicated the successful expression and purification of VP0, VP1 and VP3. The fusion purified VPs proteins produced from E. coli varied from $12-18$ $\mathrm{mg} / \mathrm{L}$ culture medium, which is equivalent with a previous report, where the three VPs genes were expressed individually in three expression vectors [17]. Forming of VP0, VP1 and VP3 is inter-state of making virus virion in their natural life cycle. VP0, VP1 and VP3, cleaved from the $\mathrm{P} 1$ protein precursor, can remain associated in a protein complex and act as a monomer for the self-assembly of five monomers into the pentameric capsid subunit [18]. The study of FMDV structural proteins were mainly focused on VP1, the most important antigen among structure capsid proteins for immunization [10,11, 19]. However, FMDV owns the ability to form empty capsids sharing the same antigenicity as natural form, but does not contain RNA [20]. Based on their natural ability, some research groups have succeed in genetic production of VLPs from baculovirus systems and test their immunity $[12,13,21]$. The initial results showed the vaccines protect tested animals from FMDV administration. 


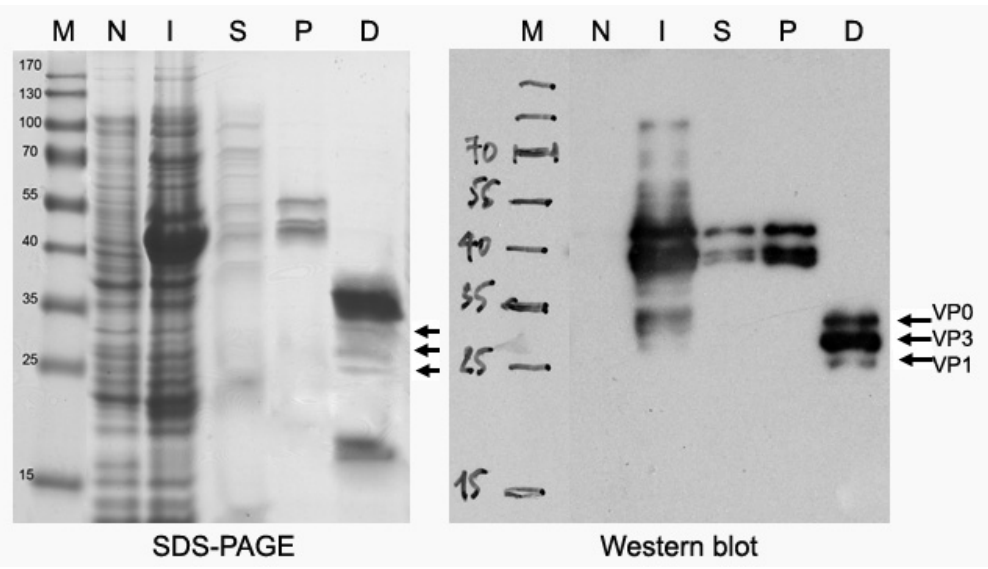

Figure 4. VPs production and immunoblotting. Three expression cassettes of VP0, VP1 and VP3 was expressed in BL21 (DE3)-RIL cells under the control of T7 promotors and IPTG induction. After 20 hours induction, the recombinant proteins were highly expressed (lane I). None-induction was showed as control (lane N). Proteins were tested for water solubility (lane S) and purified using Ni resin (lane P). VP

fusion proteins were treated with SUMO protease to separate VPs and His-SUMO tag (lane D). The

samples were loaded on $12 \%$ SDS-PAGE, stained with coomasie blue and recombinant proteins were detected by western blotting with FMDV polyclonal antibody.

Bacterial expression systems, especially in E. coli take numerous advantages over other expression systems. Most recombinant proteins can be cloned and expressed in E.coli. The use of E.coli for protein expression is well documented for its advantages of low cost, easy transformation and fermentation, and high protein yields. However, solubility may be an issue, since some proteins are insoluble and aggregate in inclusion bodies [22]. So overcome those difficulties are the fundamental steps for making VLP vaccine. Some other reported the success of making VLPs of Porcine circovirus 2 [23], influenza virus [24], hepatitis B [25], by using $E$. coli expression system. In this study, we shown that the recombinant proteins of FMDV can be expressed as the water soluble proteins, an important feature to form VLP lately.

\section{CONCLUSION}

Expression vectors pHD-Amp-VP0, pHD-Kan-VP1, pHD-Amp-VP3 and pHD-Amp-VP3VP0 were successfully designed and cloned in E. coli cells. Concurrently expression of pHDKan-VP1 and pHD-Amp-VP3-VP0 were resulted in soluble proteins with the fusions of (6HisSUMO-VP0, 6His-SUMO-VP1 and 6His-SUMO-VP3), with the expected molecular weight about 52, 48 and $45 \mathrm{kDa}$, respectively. The fusion proteins could be cleaved by His-SUMO protease to form 6His-SUMO and intrinsic VP0, VP1 and VP3. The result of immunoblotting showed that expressed protein VP0, VP1 and VP3 had molecular mass weight of $32 \mathrm{kDa}, 28$ $\mathrm{kDa}$ and $25 \mathrm{kDa}$, respectively.

Acknowledgement. This work was financial support by project: Investigation on preparing recombinant antigens in the form virus likes particles (VLP) for further vaccine production against FMDV type O, code KC.04.19/11-15 and mutual fund from Dr. Ting-Fang Wang's lab. 


\section{REFERENCES}

1. Parida S. - Vaccination against foot-and-mouth disease virus: strategies and effectiveness, Expert Rev Vaccines 8 (3) (2009) 347-65.

2. Le V. P., Le V. P., Nguyen T., Park J. H., Kim S. M., Ko Y. J., Lee H. S., Nguyen V. C., Mai T. D., Do T. H., Cho I. S., Lee K. N. - Heterogeneity and genetic variations of serotypes $\mathrm{O}$ and Asia 1 foot-and-mouth disease viruses isolated in Vietnam, Vet Microbiol 145 (3-4) (2010) 220-9.

3. Le V. P., Nguyen T., Lee K. N., Ko Y. J., Lee H. S., Nguyen V. C., Mai T. D., Do T. H., Kim S. M., Cho I. S., Park J. H. - Molecular characterization of serotype A foot-andmouth disease viruses circulating in Vietnam in 2009, Vet. Microbiol. 144 (1-2) (2010) 58-66.

4. Carrillo C., Tulman E. R., Delhon G., Lu Z., Carreno A., Vagnozzi A., Kutish G. F., Rock D. L. - Comparative genomics of foot-and-mouth disease virus, J. Virol. 79 (10) (2005) 6487-504.

5. Jamal S. M. and G. J. Belsham - Foot-and-mouth disease: past, present and future, Vet. Res. 44 (2013) 116.

6. Goodwin S., Tuthill T. J., Arias A., Killington R. A., Rowlands D. J. - Foot-and-mouth disease virus assembly: processing of recombinant capsid precursor by exogenous protease induces self-assembly of pentamers in vitro in a myristoylation-dependent manner, J. Virol. 83 (21) (2009) 11275-82.

7. Doel T. R. - FMD vaccines, Virus Res. 91 (1) (2013) 81-99.

8. Cox S. J., Voyce C., Parida S., Reid S. M., Hamblin P. A., Paton D. J., Barnett P. V. Protection against direct-contact challenge following emergency FMD vaccination of cattle and the effect on virus excretion from the oropharynx, Vaccine 23 (9) (2005) 110613.

9. Rodriguez L. L. and M. J. Grubman - Foot and mouth disease virus vaccines, Vaccine 27 (4) (2009) D90-4.

10. Alam S. M., Ruhul A., Mohammed Z., Anwar H., and Munawar S. - Antigenic heterogeneity of capsid protein VP1 in foot-and-mouth disease virus (FMDV) serotype Asia 1, Adv. Appl. Bioinform. Chem. 6 (2013) 37-46.

11. Wang J. H., Liang C. M., Peng J. M., Shieh J. J., Jong M. H., Lin Y. L., Sieber M., Liang S. M. - Induction of immunity in swine by purified recombinant VP1 of foot-and-mouth disease virus, Vaccine 21 (25-26) (2003) 3721-9.

12. Cao Y., Sun P., Fu Y., Bai X., Tian F., Liu X., Lu Z., Liu Z. - Formation of virus-like particles from O-type foot-and-mouth disease virus in insect cells using codon-optimized synthetic genes, Biotechnol Lett. 32 (9) (2010) 1223-9.

13. Mohana Subramanian B., Madhanmohan M., Sriraman R., Chandrasekhar Reddy R. V., Yuvaraj S., Manikumar K., Rajalakshmi S., Nagendrakumar S. B., Rana S. K., Srinivasan V. A. - Development of foot-and-mouth disease virus (FMDV) serotype O virus-likeparticles (VLPs) vaccine and evaluation of its potency, Antiviral Res. 96 (3) (2012) 28895. 
14. Lee C. D., Sun H. C., Hu, S. M., Chiu, C. F., Homhuan, A., Liang, S. M., Leng, C. H., Wang, T. F. - An improved SUMO fusion protein system for effective production of native proteins, Protein Sci. 17 (7) (2008) 1241-8.

15. Nguyen Phuong Hoa, N. H. D., Vu Thi Thu Huyen, Le Thi Hong Minh, Nguyen Thi Kim Cuc, Ton That Huu Dat, Le Van Phan, Pham Viet Cuong - Variation in genteic structure of the genes coding for envelope protein of vietnamese foot and mouth disease viruses, Vietnam Journal of Chemistry 53 (2e) (2015) 178-182.

16. Zeng G. - Sticky-end PCR: new method for subcloning, Biotechniques 25 (2) (1998) 206-8.

17. Guo H. C., Sun S. Q., Jin Y., Yang S. L., Wei Y. Q., Sun D. H., Yin S. H., Ma J. W., Liu Z. X., Guo J. H., Luo J. X., Yin H., Liu X. T., Liu D. X. - Foot-and-mouth disease viruslike particles produced by a SUMO fusion protein system in Escherichia coli induce potent protective immune responses in guinea pigs, swine and cattle, Vet Res. 44 (2013) 48.

18. Knipe T., Rieder E., Baxt B., Ward G., Mason P. W. - Characterization of synthetic footand-mouth disease virus provirions separates acid-mediated disassembly from infectivity, J. Virol. 71 (4) (1997) 2851-6.

19. Baxt B., Morgan D. O., Robertson B. H., Timpone C. A. - Epitopes on foot-and-mouth disease virus outer capsid protein VP1 involved in neutralization and cell attachment, $\mathrm{J}$. Virol. 51 (2) (1984) 298-305.

20. Rweyemamu M. M., G. Terry, and T. W. Pay - Stability and immunogenicity of empty particles of foot-and-mouth disease virus, Arch. Virol. 59 (1-2) (1979) 69-79.

21. Guo C., Zhang C., Zheng H., Huang Y. - Recombinant adenovirus expression of FMDV P1-2A and 3C protein and its immune response in mice, Res. Vet. Sci. 95 (2) (2013) 736-41.

22. Chen R. - Bacterial expression systems for recombinant protein production: E. coli and beyond, Biotechnol Adv. 30 (5) 2012) 1102-7.

23. Yin S., Sun S., Yang S., Shang Y., Cai X., Liu X. - Self-assembly of virus-like particles of porcine circovirus type 2 capsid protein expressed from Escherichia coli, Virol. J. 7 (2010) 166.

24. Jegerlehner A., Zabel F., Langer A., Dietmeier K., Jennings G. T., Saudan P., Bachmann M. F. - Bacterially produced recombinant influenza vaccines based on virus-like particles. PLoS One 8(11) (2013) p. e78947.

25. Holmes K., Shepherd D. A., Ashcroft A. E., Whelan M., Rowlands D. J., Stonehouse N. J. - Assembly Pathway of Hepatitis B Core Virus-like Particles from Genetically Fused Dimers, J. Biol. Chem. 290 (26) (2015) 16238-45. 